\title{
Erratum to "Assessment of solid components of borderline ovarian tumor and stage I carcinoma: added value of combined diffusion- and perfusion-weighted magnetic resonance imaging"
}

\section{See Hyung Kim}

Department of Radiology, School of Medicine, Kyungpook National University, Kyungpook National University Hospital, Daegu, Korea

Received: March 11, 2020

Accepted: March 17, 2020

Corresponding author:

See Hyung Kim

Department of Radiology, School of Medicine, Kyungpook National University, Kyungpook National University Hospital, 130 Dongdeokro, Jung-gu, Daegu 41944, Korea

Tel: +82-53-200-5390

Fax: +82-53-422-2677

E-mail: kimseehyung72@outlook.kr
In the article entitled "Assessment of solid components of borderline ovarian tumor and stage I carcinoma: added value of combined diffusion- and perfusion-weighted magnetic resonance imaging" [1], the affiliation for the corresponding author was incorrectly listed as Kyungpook National University. It has been changed to "Kyungpook National University, Kyungpook National University Hospital".

\section{Before correction}

Department of Radiology, School of Medicine, Kyungpook National University, Daegu, Korea

\section{After correction}

Department of Radiology, School of Medicine, Kyungpook National University, Kyungpook National University Hospital, Daegu, Korea

\section{Acknowledgments}

\section{Conflicts of interest}

No potential conflict of interest relevant to this article was reported.

\section{ORCID}

See Hyung Kim, https://orcid.org/0000-0002-3268-3091

\section{References}

1. Kim SH. Assessment of solid components of borderline ovarian tumor and stage I carcinoma: added value of combined diffusion- and perfusion-weighted magnetic resonance imaging. Yeungnam Univ J Med 2019;36:231-40. 